\title{
WEIGHT LOSS WITH BARIATRIC SURGERY IMPROVES ISCHAEMIA MODIFIED ALBUMIN LEVELS AND OXIDATIVE STATUS IN MORBIDLY OBESE PATIENTS \#EP-555
}

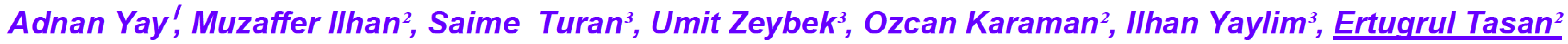

Bezmialem University, Department of Internal Medicine, Istanbul, TURKEY

${ }^{2}$ The Institute of Experimental Medicine, Department of Molecular Medicine, İstanbul University, TURKEY

${ }^{3}$ Bezmialem University, Department of Endocrinology and Metabolism, Istanbul, TURKEY

\section{OBJECTIVES}

Oxidative stress is one of the possible mechanisms involved in the pathogenesis of the obesity-related metabolic complication. In this study, we aimed to investigate effect of weight loss with bariatric surgery on IMA, hsCRP (high sensitive C-reactive protein), serum total antioxidant status (TAS), total oxidant status (TOS) levels.

\section{METHODS}

In this prospective study, plasma IMA, hs-CRP, TAS and TOS levels were measured before and 6 months after laparoscopic sleeve gastrectomy in 60 morbidly obese patients with a body mass index (BMI) of $46,6 \pm 6,7 \mathrm{~kg} / \mathrm{m}^{2} .43$ age-matched healthy subjects with a $\mathrm{BMI}$ of $24,6 \pm 3$ recruited as controls. Metabolic parameters including serum levels of fasting glucose, $\mathrm{HbA1c}$, homeostatic model assessment of insulin resistance (HOMA-IR), C-peptide, LDL, HDL, triglyceride were also measured before and after surgery.

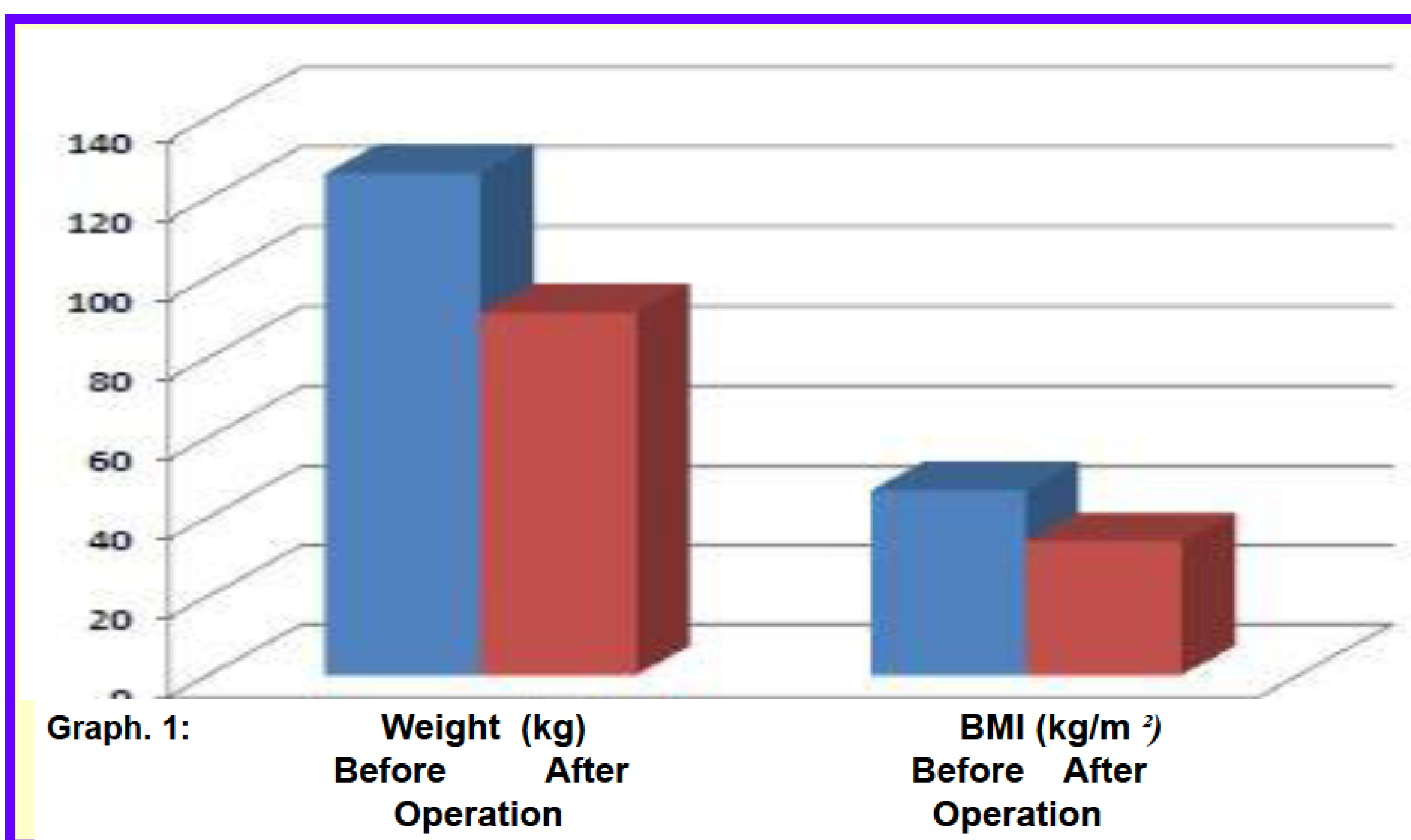

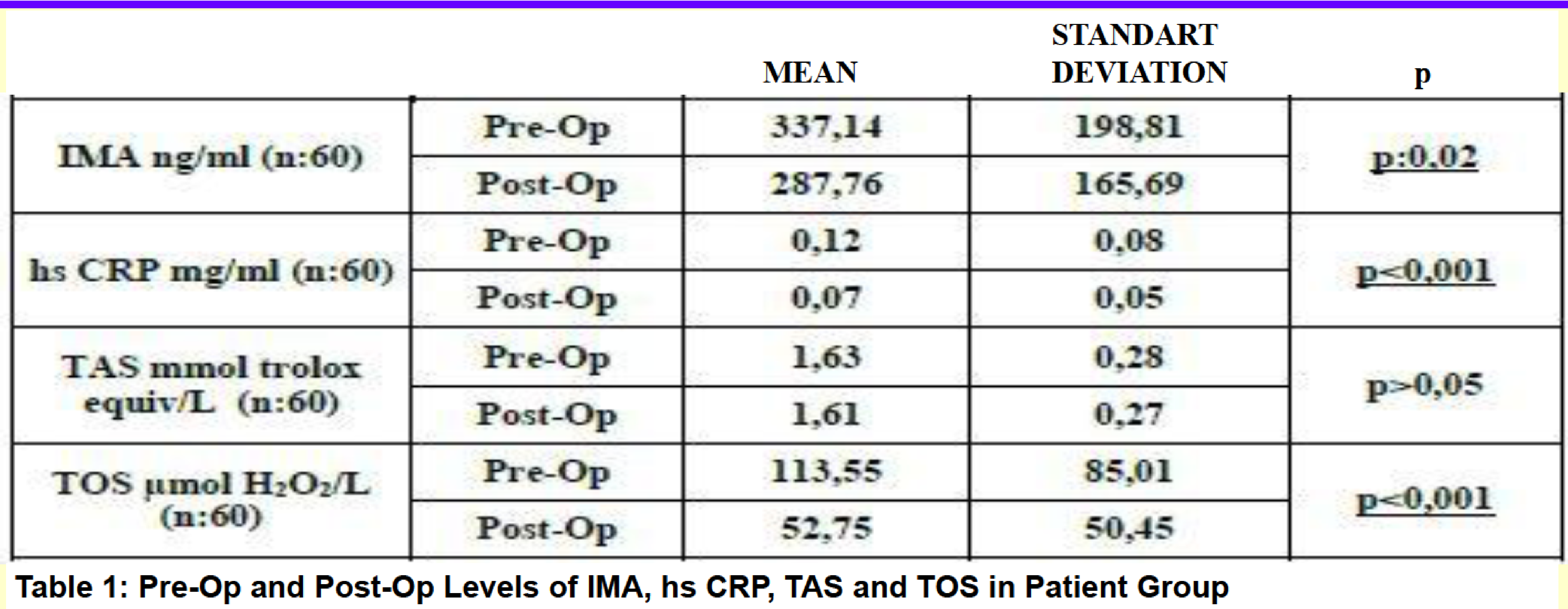

RESULTS

Excess weight loss (EWL) and percentage of EWL were $34,9 \pm 10,5 \mathrm{~kg}$ and $\% 27,8$ in obese patients. Plasma IMA levels before surgery was significantly increased in patients compared to controls $(p<0,05)$. We found significantly increased hsCRP levels in patients compared to controls $(p<0,001)$. Plasma IMA and hs-CRP levels were significantly decreased in patients after six month of bariatric surgery ( $p<0,05$ and $p<0,001$, respectively). At sixth month of surgery, BMI of patients was still significantly higher than controls $(p<0,01)$, hs-CRP levels were significantly increased in patients $(p<0,001)$ but there was no significant difference in IMA levels. A statistically significant reduction was found in the TOS values after the surgery $(p<0,001)$, but there were no significant changes in the TAS levels (Table 1). A significant reduction in BMI and total weight has showed in Graph 1. Significantly decreased levels of fasting glucose $(p<0,01), H b A 1 c(p<0,001)$, HOMAIR $(p<0,01)$, C-Peptide $(p<0,001)$, LDL-C $(p<0,001)$ and triglyceride $(p<0,001)$ and increased levels of HDL-C ( $<<0,001)$ were observed after bariatric surgery.

\section{CONCLUSIONS}

Our findings supported that weight loss with bariatric surgery improves IMA, hs-CRP, TOS levels and metabolic profile in patients. Along with still higher BMI in patients after surgery, no significant difference in IMA levels between obese and healthy group suggested that bariatric surgery could have beneficial effects on oxidative stress beyond weight loss. 